\title{
How can collaboration be strengthened between public health and primary care? A Dutch multiple case study in seven neighbourhoods
}

Ilse Storm ${ }^{1 *}$, Anke van Gestel $l^{2}$, len van de Goor ${ }^{3}$ and Hans van Oers ${ }^{3,4}$

\begin{abstract}
Background: Although public health and primary care share the goal of promoting the health and wellbeing of the public, the two health sectors find it difficult to develop mutually integrated plans and to collaborate with each other. The aim of this multiple case study was to compare seven neighbourhoods in which a stepwise approach based on two central tools (district health profile and policy dialogue) was used to develop integrated district plans and promote collaboration.
\end{abstract}

Methods: The stepwise approach involved the following steps: 1 Getting to know the neighbourhood, 2 Assembling the workgroup, 3 Analysing the neighbourhood, 4 Developing a district health profile, 5 Preparing policy dialogue, 6 Holding local dialogues, 7 Embedding integrated district plans and collaboration. To supervise this process, a core team was assembled for each neighbourhood, consisting of people drawn from both public health and primary care. Both the use of the two tools and the collaboration were studied by means of documentary analysis, interviews, questionnaires and observations.

Results: The seven neighbourhoods differed in the way the two tools of the stepwise approach were used: general versus focused profiles, the actors involved, the aims of the dialogue or the intensity of the steps. There were also similarities: profile indicators (e.g., population prognosis, vulnerability) and dialogue themes (e.g., obesity, social cohesion). The local actors experienced that the combination of both tools facilitates the process of bringing public health and primary care closer together, and that it is essential to invest sufficiently in the integration of profile data and in involving appropriate actors in the dialogue (e.g., GPs, residents). Collaboration was perceived as positive (e.g., feels involved, focus on consensus), but a starting process. Local actors also believe that the stepwise approach supported the process.

Conclusion: A stepwise approach involving the combined use of district health profiles and policy dialogues promotes the integrated planning of health activities and facilitates collaboration between public health and primary care at the local level. Local differences may arise in the intensity and form of the various steps, but because they are practical and clearly defined, they remain transferrable to other neighbourhoods.

Keywords: Collaboration, Public health, Primary care, District health profiles, Policy dialogues, Integrated health policy, Stepwise approach

\footnotetext{
* Correspondence: ilse.storm@rivm.nl

${ }^{1}$ National Institute for Public Health and the Environment, Centre for Health and Society, PO Box 13720 BA, Bilthoven, The Netherlands

Full list of author information is available at the end of the article
} 


\section{Background}

In the Netherlands, the importance of collaboration between public health and primary care at the neighbourhood level is emphasised in the government policy document Health nearby [1, 2]. The WHO Regional Office for Europe stressed the linkages between these health sectors in the briefing paper Health 2020: A European policy framework and strategy for the $21^{\text {st }}$ century [3]. Collaboration between public health and primary care facilitates the improvement of health standards and is supposed to have health benefits for the local population [4-7]. Although public health and primary care share the goal of promoting the health and wellbeing of the public, the two sectors find it difficult to develop mutually integrated plans and to collaborate with each other $[6,8]$. At the neighbourhood level this often leads to differing community diagnoses with differing priorities, and consequently poorly integrated or even conflicting health plans or activities. Literature shows that synergy could emerge with regard to planning health services according to population characteristics and needs, advocacy for healthy communities, equity and access, clinical early preventive intervention and clinical promotion of healthy lifestyle [9]. Integrated plans and collaboration can be facilitated by the use of tools or strategies. However, few practical developed tools for collaboration between public health and primary care at local level are described [10, 11].

One of the preconditions for effective collaboration is the integrated collection of descriptive statistical information about health, preventive interventions and care in the neighbourhood, by means of a district health profile [12]. A district health profile is a statistical report on the neighbourhood and its residents, concerned with health and health determinants [13]. In local practice, there are often separate care-focused profiles (e.g., neighbourhood profiles based on primary care registries) or public health-focus health profiles (e.g., health profiles based on health surveys by regional public health services) [13-17]. The joint preparation of a district health profile that considers issues from various angles promotes mutual familiarity, shared focus and collaboration between public health and primary care. A second precondition is creating a dialogue between the relevant stakeholders in the neighbourhood. A policy dialogue is a tool involving a process characterised by one or more debates, by means of which technical knowledge, stakeholders' knowledge and residents' knowledge are integrated $[18,19]$. Such a dialogue can contribute to cohesive planning or policy development for a neighbourhood, by increasing insight into locally significant issues, introducing local actors to each other and promoting post-dialogue willingness to plan and implement initiatives [11]. By combining the joint preparation of a district health profile with a policy dialogue between the relevant actors in the neighbourhood aimed at setting priorities, it is supposed that an integrated district plan or policy tailored to the local situation can be developed $[10,20]$. By doing so in relatively small geographical and organisational areas (i.e., neighbourhoods, districts), it is possible to promote the optimal use of existing local networks and expertise, and to encourage public participation [1].

The strategy of regional public health services, primary healthcare providers, local government and the public collectively developing district health plans by a process of integrated district health profiling and policy dialogue involving actors with a variety of perspectives is innovative within the public health and primary care field [11]. The aim of this multiple case study was to compare seven neighbourhoods in which a stepwise approach based on two central tools (district health profile and policy dialogue) was used to develop integrated district plans and promote collaboration. The research questions addressed by the study were: 1 . How are the two tools (district health profile and policy dialogue) used to develop integrated district health plans or activities and to promote collaboration?, 2. How is the use of the two tools as well as the collaboration process viewed by local actors? The insights yielded by this multiple-case study may be useful to parties seeking to promote collaboration between public health and primary care to secure health benefits for local communities.

\section{Methods}

\section{Study-design}

For this study a multiple case design is used, in which several cases and qualitative methods are taken into account $[21,22]$. The study sample consisted of seven cases, each case being a neighbourhood in the province (region) of Noord-Brabant. In this context, a neighbourhood is defined as an operationally viable geographical or organisational area (e.g., part of municipality, village) [23]. The cases were selected by managers of three different regional public health services (GGDs) and one regional primary care support structure (ROS Robuust) which falls under the control of the provincial authorities [24, 25]. They selected the neighbourhoods mainly on the basis of existing contacts, open attitude for collaboration between public health and primary care (e.g., GP and municipality), location (at least two municipalities of each participating regional public health service) and size (two medium-sized and five small municipalities). The province contains 67 municipalities [26]. In each neighbourhood a stepwise approach to develop integrated district plans or activities was implemented in the period July 2013 to July 2014 (roughly a year). To supervise the process, a core team was assembled for 
each neighbourhood, consisting of an epidemiologist and a policy advisor from the regional public health services (GGD) and a policy advisor from the regional primary care support structure (ROS). To help them with their work, core team members attended three inspiration days' (devoted to collaborating and networking in the neighbourhood, preparing and holding policy dialogues, and embedding plans or activities) and materials were made available (e.g., specimen district health profiles and policy dialogue work forms). A project group concerned in the province and a steering committee consisting of experts in the fields of public health, health care, the primary care association, health insurance companies, advocacy citizens, municipalities, health centres and knowledge institutes advised on this multiple-case study.

\section{A stepwise process approach}

In each of seven neighbourhoods, a stepwise approach was used to develop an integrated district plan or activities, focusing on collaboration between public health and primary care with a view to improving the health of the local population. District health profiles and policy dialogues were the central tools used for the approach. The stepwise approach is summarised in Fig. 1.
The stepwise approach for this study was developed on the basis of existing tools for district profiles and intersectoral action for health $[15,16]$, and nine exploring interviews (4 regional public health service, 2 regional primary care support structure, 2 municipalities, 1 province). These exploring interviews focused on enabling and disabling factors in working with district profiles or policy dialogues, but also on neighbourhoodfocused working and collaborating between public health and primary care in practice.

\section{Data collection}

Use of the tools (district health profiles and policy dialogues) used in the stepwise integrated district (activity) plan development process was studied by means of documentary analysis, digital questionnaires, interviews and observations. In order to answer the two research questions, data were gathered regarding both the tool content and the (collaboration) process:

- Documentary analysis of district health profile presentations, dialogue reports and district plan or activity papers (content data).

- (Group) interviews with each of the seven core teams (see Table 1). Two semi-structured interviews lasting about ninety minutes were held with each

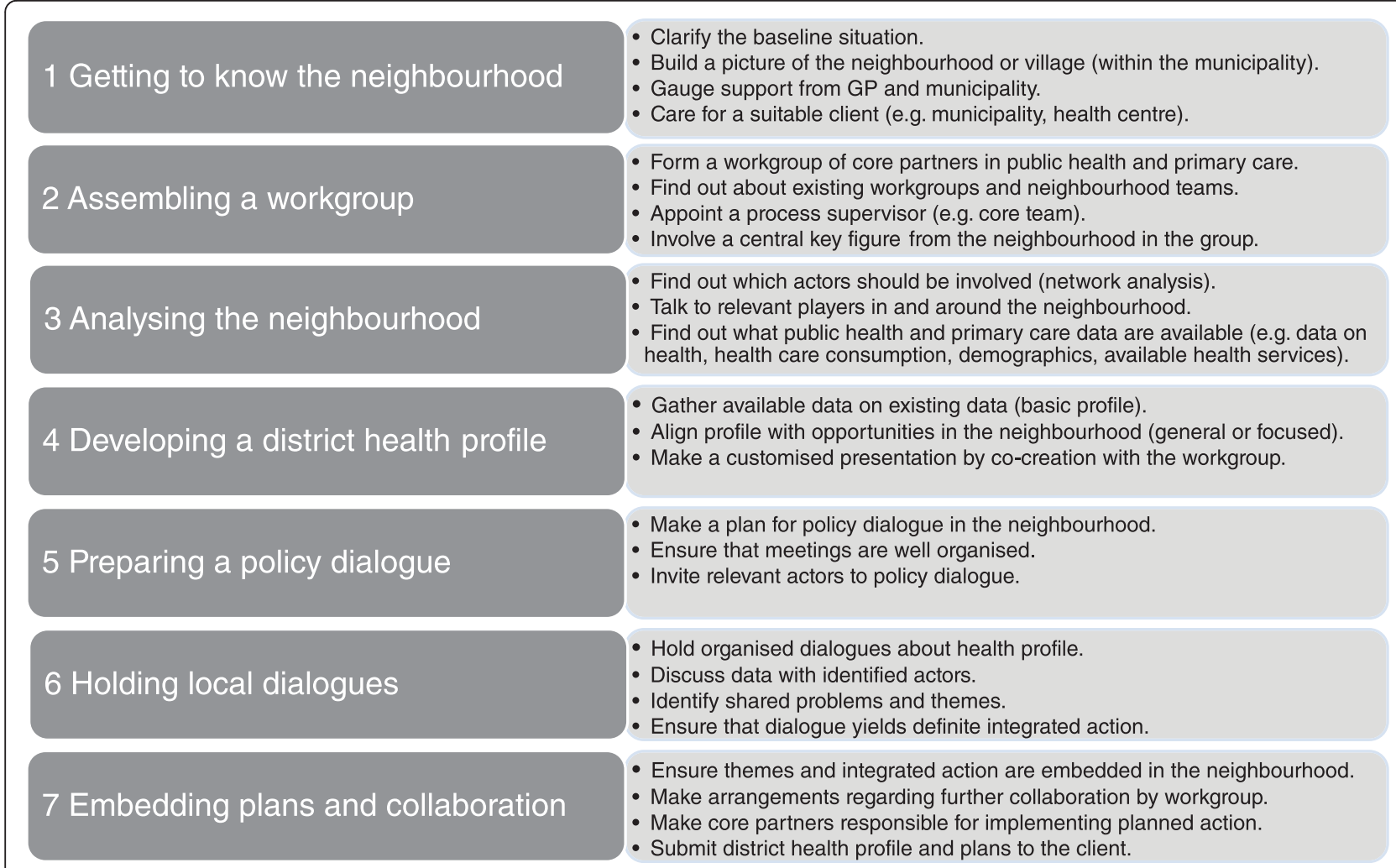

Fig. 1 Stepwise approach 
Table 1 Overview number of (group) interviews and questionnaires

\begin{tabular}{|c|c|c|c|c|}
\hline \multirow{2}{*}{$\frac{\text { Methods }}{\text { (Group) interviews }}$} & \multirow{2}{*}{$\frac{\text { Number }}{14}$} & \multicolumn{2}{|c|}{ Respondent's position or organization } & \multirow{2}{*}{$\begin{array}{l}\text { Professions respondents } \\
\text { Epidemiologist, Policy advisor public health }\end{array}$} \\
\hline & & Core team & $\begin{array}{l}\text { Regional Public Health } \\
\text { Services }\end{array}$ & \\
\hline & & & $\begin{array}{l}\text { Regional Primary care } \\
\text { support structure }\end{array}$ & Policy advisor primary care ( $2 \times 7$ groups interviews) \\
\hline & 4 & Managers core team & $\begin{array}{l}\text { Regional Public Health } \\
\text { Services }\end{array}$ & Managers \\
\hline & & & $\begin{array}{l}\text { Regional Primary care } \\
\text { support structure }\end{array}$ & \\
\hline & (18 total) & & & \\
\hline \multirow[t]{7}{*}{ Online questionnaires } & 16 & Core team & $\begin{array}{l}\text { Regional Public Health } \\
\text { Services }\end{array}$ & Epidemiologist (6x), Policy advisor public health (5x) \\
\hline & & & $\begin{array}{l}\text { Regional Primary care } \\
\text { support structure }\end{array}$ & Policy advisor primary care $(5 x)$ \\
\hline & 24 & Relevant local actors & Municipality & $\begin{array}{l}\text { Social Support Act officer (1x), Policy officer Public } \\
\text { Health }(1 \mathrm{x}) \text {, Municipal neighbourhood work } \\
\text { coordinator }(1 \mathrm{x})\end{array}$ \\
\hline & & & Welfare organization & Social workers $(2 x)$ \\
\hline & & & Primary care & $\begin{array}{l}\text { Dieticians/coach ( } 2 x) \text {, Neighbourhood nurses }(2 x) \\
\text { GP's }(2 x) \text {, Manager }(2 x) \text {, Manager home care }(1 x)\end{array}$ \\
\hline & & & Residents & $\begin{array}{l}\text { Retired manager }(1 \mathrm{x}) \text {, Member residents platform }(2 \mathrm{x}) \text {, } \\
\text { Journalist/inhabitant }(1 \mathrm{x}) \text {, Officer elderly people's } \\
\text { organization }(1 \mathrm{x})\end{array}$ \\
\hline & (40 total) & & Other organisations & $\begin{array}{l}\text { Village support worker }(1 \mathrm{x}) \text {, Professional disability care } \\
\text { housing association }(2 \mathrm{x}) \text {, Police officer }(1 \mathrm{x}) \text {, Officer } \\
\text { education }(1 \mathrm{x})\end{array}$ \\
\hline
\end{tabular}

core team, one partway through the process (after step 3) and one at the end (during step 7). Hence, two sets of seven interviews took place. In addition, partway through the process, an interview was held with each of the managers of the seven core teams, because of the role that they played in starting up the process in the neighbourhoods (e.g., contacting municipal councillors and municipal officials). As some managers were responsible for two teams in total four interviews were held. A total of eighteen interviews was carried out, focusing on the tools, the process steps and collaboration between public health and primary care. The interviews were all recorded using a digital voice recorder and transcribed (content and process data).

- Online questionnaires completed both by the core teams and by relevant local actors (see Table 1 ). Relevant partners are partners in the field or municipality, primary health care professionals, social welfare professionals and local residents. A total of fifty-one actors from six pilot neighbourhoods were approached and forty of them returned the questionnaire (a response rate of $78 \%$ ). The forty respondents included sixteen of the eighteen core team members (89\%) and twenty-four of the thirty-three relevant actors (73\%). In one of the seven neighbourhoods no digital questionnaires were sent out, because this neighbourhood was significantly behind the others in terms of progression of the process. The questionnaire was made up of questions with closed answer categories (no, more no than yes, neutral, more yes than no, yes) about the district health profile, the policy dialogue and the collaboration between public health and primary care. Respondents were also asked to rate (by giving marks ranging from 1 to 10) both tools (content and process data).

- Observations at policy dialogue sessions. Two members of the research team attended nine policy dialogue sessions to obtain an impression of how the district health profile was used in the dialogue and to identify any links between the public health sector and the primary care sector that might have developed. In a number of neighbourhoods, several policy dialogues were held. The research team members recorded their observations in a report (process data).

In the interviews and digital questionnaires, the questions regarding the tools were based on McMaster University's evaluation of policy dialogues [27]. The process-related questions were based on the Intersectoral Collaboration Checklist (e.g., collaboration) and the evaluation questions of the Regional Public Health Reporting 
(e.g., content, use and usability [28-31]. The questions were developed specifically for this multiple-case study. Practical implementation of the plans and collaboration was due to take place after the project ended and was not therefore studied.

Participants in the interviews were informed that contributions included in the results would be made anonymous. The statements were also to be presented as group results and would not be reducible to individuals. On the basis of these conditions and prior to the execution of the interviews recorded on tape, participants agreed to take part and gave verbal informed consent to use the results in publications. This study was not subject to the Dutch Medical Research Involving Human Subjects Act. That means research activities including human participants is exempted from ethics approval in case they do not meet the criterion that participants are subjected to (invasive or bothersome) procedures or are required to follow rules of behaviour.

\section{Data-analysis}

In order to assess how the tools were used in the cases (research question 1), various aspects of each district health profile (e.g., area level, reference field, public health and primary care indicators, sources and presentation form) and policy dialogues (e.g., group size, dialogue work form, actors involved in the neighbourhood and chosen themes) were analysed. The concrete activities or plans and collaboration agreements arising from use of the tools were also included in the analysis. Information on similarities and differences between the seven neighbourhoods regarding the various aspects was based on analysis of data from documents, interviews and digital questionnaires.

In the assessment of how local actors viewed the stepwise approach in the cases (research question 2), various aspects of the two tools and collaboration were analysed. Where the tools were concerned, those aspects were the mean marks for the tools, the value of the profile in the policy dialogue (e.g., comprehensible presentation, relevant data), the value of the dialogue in the discussion of problems, priorities or solutions (e.g., defining themes, identifying solutions, use of results after dialogue). Based on experiences of the cases, also the dos and don'ts associated with the use of the tools were included in the analysis. Where the collaboration was concerned, the aspects were the extent to which the stepwise approach had contributed to strengthening bridges between sectors (e.g., interest in participation, appropriate actors involved, satisfied with participants' input, consensus about focus, ties between sectors) and the appetite for continued collaboration. That was done for the seven neighbourhoods by analysis of data from the interviews, questionnaires and observations. The data analyses were performed by two researchers.

\section{Results}

Use of district health profiles and policy dialogues

Tables 2 and 3 summarise the main findings regarding the use of district health profiles and policy dialogues in the seven neighbourhoods. Table 4 summarises the findings regarding the practical products of the tools' use (activities and collaboration).

\section{District health profiles}

The content and form of the district health profiles differed from neighbourhood to neighbourhood (e.g., general or focused). Indicators included in almost all profiles were population age structure, population prognosis, health status and socioeconomic vulnerability. The indicators were reflected in the profile primarily by sourcing data from the public health services and primary care support structure and, to a lesser extent, other actors, such as the municipality or health centres. In the profile presentation, a number of neighbourhoods compared neighbourhood data against data on the whole municipality, region or country (e.g., interpretation of data). The profile was presented in the form of a PowerPoint presentation or factsheet.

\section{Policy dialogues}

The diversity of the group invited to participate in the policy dialogues differed from neighbourhood to neighbourhood. In this context, 'diversity' means the extent to which not only public health and primary care actors, but also other actors (e.g., residents, police and welfare workers) were involved. The dialogues had various aims: discussing the data, inventorying wishes, exchanging ideas about issues and solutions, and selecting themes (e.g., by using a 'world café', roundtable talks and thematic meetings) [20]. In a number of neighbourhoods, several dialogues were held. Most neighbourhoods organised preparatory consultations with various parties (e.g., GP, social area team, elderly people's association) prior to the dialogue itself. Themes that were discussed in all neighbourhoods were: 1 . Obesity, 2 . Social cohesion and 3. Loneliness of the elderly.

\section{Integrated plans or activities and collaboration}

The integrated planning of activities in the seven neighbourhoods was the result of the process using the two tools. It related mainly to the themes that were brought up in the dialogues. Examples of activities on the theme of obesity include GPs teaming up with physiotherapists, the regional public health service approaching parents about an exercise programme and fruit at school, the regional public health service starting a neighbourhood campaign with local people, sports clubs and private actors. Also agreements on collaboration were made. Definite arrangements were made within the seven 
Table 2 Summary of district profile findings

\begin{tabular}{|c|c|c|c|c|c|c|c|}
\hline Neighbour-hood & Gemert & Achtse Barrier & $\begin{array}{l}\text { Heeswijk-Dinther and } \\
\text { Loosbroek }\end{array}$ & Boxtel-Oost & $\begin{array}{l}\text { Gesworen Hoek and } \\
\text { Huibeven }\end{array}$ & Banakkers & Terheijden \\
\hline \multicolumn{8}{|c|}{ Integrated district health profile } \\
\hline Area (level) & $\begin{array}{l}\text { Combination of 4-digit } \\
\text { postcode area }\end{array}$ & 4-digit postcode area & 4-digit postcode area & $\begin{array}{l}\text { 4-digit postcode } \\
\text { area }\end{array}$ & 4-digit postcode area & $\begin{array}{l}\text { 4-digit postcode } \\
\text { area/regional data }\end{array}$ & $\begin{array}{l}\text { 4-digit postcode } \\
\text { area/regional data }\end{array}$ \\
\hline $\begin{array}{l}\text { Residents } \\
\text { neighbourhood }\end{array}$ & 15.800 & 12.500 & 9.400 & 9.300 & 9.700 & 3.400 & 6.300 \\
\hline $\begin{array}{l}\text { Municipality } \\
\text { (residents in 2014) }\end{array}$ & Gemert-Bakel (29.361) & Eindhoven (221.402) & Bernheze (29.717) & Boxtel (30.356) & Tilburg (211.726) & Etten-Leur (42.395) & Drimmelen (26.671) \\
\hline $\begin{array}{l}\text { Reference area to } \\
\text { interpret profile } \\
\text { findings }\end{array}$ & $\begin{array}{l}\text { Municipality, regional } \\
\text { public health services }\end{array}$ & Municipality & $\begin{array}{l}\text { Municipality, regional } \\
\text { public health services, } \\
\text { the Netherlands }\end{array}$ & $\begin{array}{l}\text { Municipality, } \\
\text { regional public } \\
\text { health services, } \\
\text { the Netherlands }\end{array}$ & $\begin{array}{l}\text { Municipality, regional } \\
\text { public health services, } \\
\text { the Netherlands }\end{array}$ & $\begin{array}{l}\text { Municipality, regional } \\
\text { public health services, } \\
\text { the Netherlands }\end{array}$ & $\begin{array}{l}\text { Municipality, the } \\
\text { Netherlands }\end{array}$ \\
\hline $\begin{array}{l}\text { Topics covered } \\
\text { by district health } \\
\text { profile }\end{array}$ & $\begin{array}{l}\text { Population age } \\
\text { structure and prognosis; } \\
\text { socioeconomic } \\
\text { vulnerability; nature } \\
\text { and quality of human } \\
\text { environment; health } \\
\text { and wellbeing; care } \\
\text { and assistance; } \\
\text { self-sufficiency and } \\
\text { vulnerability; } \\
\text { participation and } \\
\text { loneliness }\end{array}$ & $\begin{array}{l}\text { Population age } \\
\text { structure and } \\
\text { prognosis; } \\
\text { socioeconomic } \\
\text { vulnerability; nature } \\
\text { and quality of human } \\
\text { environment; health } \\
\text { and wellbeing; care } \\
\text { and assistance; } \\
\text { self-sufficiency } \\
\text { and vulnerability; } \\
\text { participation and } \\
\text { loneliness }\end{array}$ & $\begin{array}{l}\text { Population age } \\
\text { structure and } \\
\text { prognosis; } \\
\text { socio-cultural } \\
\text { characteristics; human } \\
\text { environment; health; } \\
\text { lifestyle; quality of life; } \\
\text { spiritual dimension; } \\
\text { self-sufficiency, } \\
\text { participation and } \\
\text { support }\end{array}$ & $\begin{array}{l}\text { Population age } \\
\text { structure and } \\
\text { prognosis; } \\
\text { socio-cultural } \\
\text { characteristics; } \\
\text { physical } \\
\text { environment; } \\
\text { demand for care; } \\
\text { diseases and } \\
\text { conditions; lifestyle; } \\
\text { quality of life; } \\
\text { participation } \\
\text { and loneliness }\end{array}$ & $\begin{array}{l}\text { Population age } \\
\text { structure and } \\
\text { prognosis; environment; } \\
\text { participation; demand } \\
\text { for care; quality of life; } \\
\text { lifestyle }\end{array}$ & $\begin{array}{l}\text { Population age structure; } \\
\text { obesity; exercise; fruit \& } \\
\text { vegetable consumption; } \\
\text { GP contacts psychical } \\
\text { and social problems; } \\
\text { loneliness }\end{array}$ & $\begin{array}{l}\text { Population age } \\
\text { structure } \\
\text { and prognosis; } \\
\text { socio-cultural; } \\
\text { obesity; lifestyle; } \\
\text { physical environment; } \\
\text { social environment/ } \\
\text { surroundings; care } \\
\text { provision; demand } \\
\text { for care; diseases } \\
\text { and conditions; } \\
\text { quality of life; } \\
\text { participation }\end{array}$ \\
\hline Sources & $\begin{array}{l}\text { Regional public health } \\
\text { services }{ }^{\mathrm{a}} \text {; Regional } \\
\text { primary care support } \\
\text { structure }^{\mathrm{b}} \text { Statistics } \\
\text { Netherlands'; } \\
\text { Registrations living } \\
\text { conditions; The } \\
\text { National Institute for } \\
\text { Social Researche; } \\
\text { Municipal and health } \\
\text { centre records }\end{array}$ & $\begin{array}{l}\text { Regional public health } \\
\text { services }{ }^{\mathrm{a}} \text {; Regional } \\
\text { primary care support } \\
\text { structure }{ }^{\mathrm{b}} \text { Statistics } \\
\text { Netherlands }{ }^{\mathrm{d}} \text {; } \\
\text { Registrations } \\
\text { living conditions; } \\
\text { Health centre records }\end{array}$ & $\begin{array}{l}\text { Regional public health } \\
\text { services }^{\mathrm{a}} \text {; Regional } \\
\text { primary care support } \text { structure }^{\mathrm{b}} \text { Statistics } \\
\text { Netherlands }^{\mathrm{d}} \text {; } \\
\text { Registrations living } \\
\text { conditions; The }^{\text {National Institute for }} \\
\text { Social Research } \\
\text { National Institute for } \\
\text { Public Health and } \\
\text { Environment }^{f}\end{array}$ & 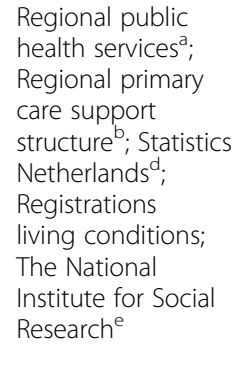 & $\begin{array}{l}\text { Regional public } \\
\text { health services } ; \\
\text { Regional primary } \\
\text { care support structure } \\
\text { Statistics Netherlands }{ }^{\text {b }} \text {; } \\
\text { Registrations living } \\
\text { conditions; The } \\
\text { National Institute } \\
\text { for Social Researche }\end{array}$ & 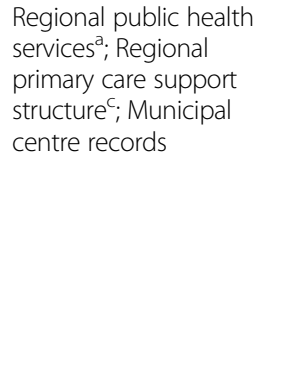 & 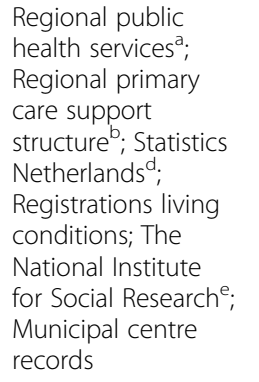 \\
\hline
\end{tabular}

aHealth surveys (GGD Monitors), ${ }^{\mathrm{b}}$ Supply and Demand Analysis Monitor (VAAM), Neighbourhood scan (ABF), Netherlands Information Network of
(LINH), ${ }^{d}$ Statistics Netherlands (CBS), ${ }^{\text {TTh }}$ The National Institute for Social Research (SCP), ${ }^{\mathrm{f}}$ National Institute for Public Health and Environment (RIVM) 
Table 3 Summary of policy dialogue findings

\begin{tabular}{|c|c|c|c|c|c|c|c|}
\hline Neighbour-hood & Gemert & Achtse Barrier & $\begin{array}{l}\text { Heeswijk-Dinther and } \\
\text { Loosbroek }\end{array}$ & Boxtel-Oost & $\begin{array}{l}\text { Gesworen Hoek and } \\
\text { Huibeven }\end{array}$ & Banakkers & Terheijden \\
\hline \multicolumn{8}{|c|}{ Policy dialogue with various parties } \\
\hline $\begin{array}{l}\text { Workgroup } \\
\text { handling } \\
\text { preparation } \\
\text { (including core } \\
\text { team }{ }^{\mathrm{a}} \text { ) }\end{array}$ & $\begin{array}{l}\text { Core team, Municipal } \\
\text { official }\end{array}$ & $\begin{array}{l}\text { Core team, GP, health } \\
\text { centre operations } \\
\text { director, manager } \\
\text { home care organisation }\end{array}$ & $\begin{array}{l}\text { Core team, Municipal } \\
\text { official }\end{array}$ & $\begin{array}{l}\text { Core team, Municipal } \\
\text { neighbourhood } \\
\text { coordinator }\end{array}$ & $\begin{array}{l}\text { Core team, Health } \\
\text { centre director, public } \\
\text { health policy officer, } \\
\text { neighbourhood } \\
\text { manager }\end{array}$ & $\begin{array}{l}\text { Core team, } \\
\text { Neighbourhood } \\
\text { manager, municipal } \\
\text { official, health } \\
\text { promotion officer }\end{array}$ & $\begin{array}{l}\text { Core team, Municipal } \\
\text { neighbourhood work } \\
\text { coordinator }\end{array}$ \\
\hline $\begin{array}{l}\text { Preparatory } \\
\text { discussions }\end{array}$ & $\begin{array}{l}\text { Short Lines workgroup } \\
\text { members (primary care, } \\
\text { welfare, municipality) }\end{array}$ & Within extended team & $\begin{array}{l}\text { Municipality, GPs, elderly } \\
\text { people's association, } \\
\text { welfare and care } \\
\text { organisations }\end{array}$ & $\begin{array}{l}\text { GP group, neighbourhood } \\
\text { body, neighbourhood nurse, } \\
\text { lay care support point }\end{array}$ & $\begin{array}{l}\text { Youth doctor/nurse, } \\
\text { primary health centre } \\
\text { professionals, } \\
\text { community school } \\
\text { steering committee }\end{array}$ & $\begin{array}{l}\text { GPs, social } \\
\text { neighbourhood team }\end{array}$ & GP and village council \\
\hline \multirow[t]{2}{*}{$\begin{array}{l}\text { Organised } \\
\text { dialogue }\end{array}$} & $\begin{array}{l}\text { 1. Presentation and } \\
\text { discussion of profile }\end{array}$ & \multirow{2}{*}{$\begin{array}{l}\text { Presentation and } \\
\text { discussion of profile, } \\
\text { theme selection }\end{array}$} & \multirow{2}{*}{$\begin{array}{l}\text { Presentation and } \\
\text { discussion of profile, } \\
\text { inventory of wishes, } \\
\text { identification of criteria } \\
\text { for theme selection }\end{array}$} & \multirow[t]{2}{*}{$\begin{array}{l}\text { Presentation and discussion } \\
\text { of profile, theme selection }\end{array}$} & \multirow{2}{*}{$\begin{array}{l}\text { Presentation and } \\
\text { discussion of profile, } \\
\text { theme selection }\end{array}$} & \multirow[t]{2}{*}{$\begin{array}{l}\text { Inventory of issues, } \\
\text { theme selection }\end{array}$} & \multirow{2}{*}{$\begin{array}{l}\text { Presentation and } \\
\text { discussion of profile, } \\
\text { inventory of wishes, } \\
\text { theme selection }\end{array}$} \\
\hline & 2. Theme selection & & & & & & \\
\hline $\begin{array}{l}\text { Number of } \\
\text { participants }\end{array}$ & 20 people & $\sim 20$ people & $\sim 45$ people & $\sim 45$ people & $\sim 25$ people & 30 people & > people \\
\hline $\begin{array}{l}\text { Neighbourhood } \\
\text { actors (examples } \\
\text { of organisations) }\end{array}$ & $\begin{array}{l}\text { Municipality (youth, } \\
\text { Social Support Act, } \\
\text { village-oriented worker), } \\
\text { GPs, physiotherapists, } \\
\text { social workers, disability } \\
\text { carers, dieticians, home } \\
\text { carers, practice support } \\
\text { workers, village support } \\
\text { worker and police }\end{array}$ & $\begin{array}{l}\text { GPs Health Centre, } \\
\text { home carers, quality } \\
\text { of life team/residents, } \\
\text { physiotherapists, } \\
\text { pharmacists, mental } \\
\text { health care officer, } \\
\text { youth health care } \\
\text { physicians of regional } \\
\text { public health services }\end{array}$ & $\begin{array}{l}\text { Care organisations, } \\
\text { schools, elderly people's } \\
\text { associations, welfare } \\
\text { workers, primary } \\
\text { professionals, volunteers, } \\
\text { Social Support Act } \\
\text { advisory board and } \\
\text { parish staff }\end{array}$ & $\begin{array}{l}\text { GPs, municipality, } \\
\text { physiotherapists, dieticians, } \\
\text { speech and language } \\
\text { therapists, Social Support Act } \\
\text { advisory board, } \\
\text { neighbourhood body, care } \\
\text { buyers, elderly people's } \\
\text { association and welfare } \\
\text { organisation }\end{array}$ & $\begin{array}{l}\text { Social workers, } \\
\text { welfare workers, } \\
\text { physiotherapists, } \\
\text { consultation and } \\
\text { training, care teams, } \\
\text { education, } \\
\text { municipality, elderly } \\
\text { people's association }\end{array}$ & $\begin{array}{l}\text { Neighbourhood } \\
\text { residents, GPs, } \\
\text { physiotherapists, } \\
\text { neighbourhood nurses, } \\
\text { social workers, } \\
\text { neighbourhood } \\
\text { association, volunteers, } \\
\text { officials and advisors }\end{array}$ & $\begin{array}{l}\text { GPs, physiotherapists, } \\
\text { residents platform, } \\
\text { village council, } \\
\text { neighbourhood } \\
\text { nurses, dieticians, care } \\
\text { organisations, sports } \\
\text { clubs, municipal } \\
\text { councillor }\end{array}$ \\
\hline $\begin{array}{l}\text { Chosen } \\
\text { theme(s) }\end{array}$ & $\begin{array}{l}\text { Loneliness, population } \\
\text { aging, poverty and } \\
\text { illiteracy, obesity, } \\
\text { exercise, complex } \\
\text { family problems }\end{array}$ & $\begin{array}{l}\text { Social cohesion/ } \\
\text { loneliness }\end{array}$ & $\begin{array}{l}\text { Social cohesion, } \\
\text { nutrition and exercise }\end{array}$ & Social cohesion/loneliness & $\begin{array}{l}\text { Poverty, obesity, } \\
\text { social cohesion }\end{array}$ & Loneliness & $\begin{array}{l}\text { Social cohesion/ } \\
\text { loneliness of the } \\
\text { elderly and adults, } \\
\text { obesity children }\end{array}$ \\
\hline
\end{tabular}


Table 4 Summary of integrated action findings

\begin{tabular}{|c|c|c|c|c|c|c|c|}
\hline Neighbour-hood & Gemert & Achtse Barrier & $\begin{array}{l}\text { Heeswijk-Dinther } \\
\text { and Loosbroek }\end{array}$ & Boxtel-Oost & $\begin{array}{l}\text { Gesworen Hoek and } \\
\text { Huibeven }\end{array}$ & Banakkers & Terheijden \\
\hline \multicolumn{8}{|l|}{$\begin{array}{l}\text { Integrated district } \\
\text { plans, activities or } \\
\text { collaboration }\end{array}$} \\
\hline \multirow[t]{4}{*}{$\begin{array}{l}\text { Content (agreement } \\
\text { on themes) }\end{array}$} & $\begin{array}{l}\text { Municipality and } \\
\text { neighbourhood } \\
\text { organisations to } \\
\text { consider what themes } \\
\text { can be included in } \\
\text { quality of life agendas } \\
\text { (e.g., loneliness, poverty, } \\
\text { obesity, illiteracy) }\end{array}$ & $\begin{array}{l}\text { More attention for } \\
\text { integration of } \\
\text { activities on the } \\
\text { theme of loneliness }\end{array}$ & $\begin{array}{l}\text { Workgroup to focus } \\
\text { on promoting social } \\
\text { cohesion, with } \\
\text { integration with } \\
\text { exercise and } \\
\text { nutrition and } \\
\text { interaction between } \\
\text { various groups. }\end{array}$ & $\begin{array}{l}\text { Welfare team to look more } \\
\text { closely at loneliness /social } \\
\text { cohesion amongst local } \\
\text { residents, neighbourhood } \\
\text { body to put question on } \\
\text { digital forum }\end{array}$ & $\begin{array}{l}\text { Poverty: Municipality to } \\
\text { organise information } \\
\text { meeting about schemes } \\
\text { for residents }\end{array}$ & $\begin{array}{l}\text { Exercise: Walking } \\
\text { club to collaborate } \\
\text { with GP. }\end{array}$ & $\begin{array}{l}\text { Obesity: GPs to work with } \\
\text { physiotherapists and } \\
\text { regional public health } \\
\text { service to approach } \\
\text { parents about an exercise } \\
\text { programme and fruit } \\
\text { at school }\end{array}$ \\
\hline & $\begin{array}{l}\text { Neighbourhood teams } \\
\text { to include themes in } \\
\text { approach plan }\end{array}$ & & $\begin{array}{l}\text { The elderly people's } \\
\text { association has } \\
\text { already started } \\
\text { forging ties } \\
\text { between } \\
\text { programmes for the } \\
\text { elderly and for } \\
\text { young people }\end{array}$ & & $\begin{array}{l}\text { Obesity: Regional Public } \\
\text { Health Service to initiate } \\
\text { coordination of existing } \\
\text { initiatives and } \\
\text { neighbourhood } \\
\text { campaign with local } \\
\text { people/sports clubs/ } \\
\text { private partners. }\end{array}$ & $\begin{array}{l}\text { Social cohesion: } \\
\text { activities in } \\
\text { Mindfulness Week }\end{array}$ & $\begin{array}{l}\text { Social cohesion and } \\
\text { loneliness: meetings to } \\
\text { be organised and a practical } \\
\text { project 'samen is leuker' ('it's } \\
\text { more fun together') with } \\
\text { the aim of highlighting } \\
\text { willingness to do volunteer } \\
\text { work }\end{array}$ \\
\hline & $\begin{array}{l}\text { Municipality to agenda } \\
\text { report with } \\
\text { recommendations in } \\
\text { Social Support Act } \\
\text { Implementation Core } \\
\text { Team }\end{array}$ & & & & $\begin{array}{l}\text { Social cohesion: } \\
\text { municipality, Regional } \\
\text { Public Health Service } \\
\text { and social workers to } \\
\text { involve residents in } \\
\text { neighbourhood and } \\
\text { problems via } \\
\text { neighbourhood lunches }\end{array}$ & $\begin{array}{l}\text { People moving into } \\
\text { the neighbourhood } \\
\text { to be welcomed } \\
\text { and contacted } \\
\text { about life events } \\
\text { (e.g., births and } \\
\text { deaths) }\end{array}$ & \\
\hline & & & & & & $\begin{array}{l}\text { Approach for } \\
\text { younger target } \\
\text { group to be } \\
\text { developed } \\
\text { (involving young } \\
\text { people, youth } \\
\text { health care and } \\
\text { other relevant } \\
\text { organisations) }\end{array}$ & \\
\hline \multirow[t]{2}{*}{$\begin{array}{l}\text { Process (agreement } \\
\text { on colla-boration) }\end{array}$} & $\begin{array}{l}\text { Meeting: municipality to } \\
\text { organise follow-up } \\
\text { meeting after six } \\
\text { months }\end{array}$ & $\begin{array}{l}\text { Meeting: workgroup } \\
\text { to organise meeting } \\
\text { of care professionals, } \\
\text { welfare and informal } \\
\text { care }\end{array}$ & $\begin{array}{l}\text { Ties: workgroup to } \\
\text { seek ways to realise } \\
\text { positive health } \\
\text { through practical } \\
\text { activities }\end{array}$ & $\begin{array}{l}\text { Ties: workgroup to seek } \\
\text { ties with existing activities } \\
\text { and ongoing projects in } \\
\text { the neighbourhood (social } \\
\text { neighbourhood team, } \\
\text { catering point, living room } \\
\text { project, generation garden) }\end{array}$ & $\begin{array}{l}\text { Ties: municipality to } \\
\text { investigate possibility of } \\
\text { subsidisation }\end{array}$ & $\begin{array}{l}\text { Ties: social } \\
\text { neighbourhood } \\
\text { team, } \\
\text { neighbourhood } \\
\text { association and GPs } \\
\text { to get to know } \\
\text { each other better }\end{array}$ & $\begin{array}{l}\text { Meeting: follow-up meeting } \\
\text { to be organised, to which } \\
\text { each professional brings a } \\
\text { Terheijden resident; } \\
\text { continuation of activities } \\
\text { related to the themes of } \\
\text { obesity and social cohesion }\end{array}$ \\
\hline & $\begin{array}{l}\text { Regional Public Health } \\
\text { Service to offer support }\end{array}$ & $\begin{array}{l}\text { Core team placed } \\
\text { item in local paper } \\
\text { inviting local }\end{array}$ & & $\begin{array}{l}\text { Communication: } \\
\text { workgroup to inform } \\
\text { residents about activities at }\end{array}$ & $\begin{array}{l}\text { Regional Public Health } \\
\text { Service wishes to } \\
\text { affiliate to 'Young }\end{array}$ & $\begin{array}{l}\text { Working visits to } \\
\text { exemplary sites }\end{array}$ & \\
\hline
\end{tabular}

Municipality to agenda report with

mplementation Core Service to offer support inviting local campaign with loca

private partners.

Social cohesion:

problems via

developed

involving young

health care an

organisations)

Meeting: workgroup Ties: workgroup to e ansionals, positive health activities

Service wishes to exemplary sites 
Table 4 Summary of integrated action findings (Continued)

to neighbourhood residents to provide

input and assistance,

yielding a number

of responses

Robuust and municipality Social map to be

to discuss result in Short produced, insight

Lines workgroup and to into activities in the

put integration of primary neighbourhood

care with social domain

on agenda

Social map: Municipality Joint website for

to produce a digital the neighbourhood

social map

initiative by health

Boxtel-Oost day and to ask people on healthy

weight/JOGG' Tilburg

and involve local

residents more

Municipality to support Meeting: municipality to Social map:

communication

organise follow-up Neighbourhood

$\begin{array}{ll}\text { organise follow-up } & \text { Neighbourhoo } \\ \text { meetings between } & \text { map to be }\end{array}$

public health and developed with

primary care

input from all

Social map: Municipality

to develop social map 
neighbourhoods for the various actors to continue meeting and sharing knowledge regarding public health, primary care and residents. In a number of neighbourhoods, the proposed activities and interactions were specified in neighbourhood plans or responsibility for them passed to existing workgroups.

\section{Experiences with the tools and collaboration}

Figures 2 and 3 summarise the views given by the core teams and other relevant actors (e.g., the municipality, welfare workers, primary care staff and local residents) regarding the tools and collaboration in the stepwise approach.

\section{Participants' views regarding the tools}

The general view was that the district health profile and the policy dialogue both have added value for the process of bringing public health and primary care closer together at the neighbourhood level. The core team members were a little more positive about both tools than the other relevant actors. Core teams experienced that the combination of the two tools was particularly beneficial with regard to the generation of both quantitative and qualitative data.

The district health profile was rated as satisfactory (7 on a scale of 1-10) by both the core teams and the actors. They thought that it was a good starting point for discussion regarding issues and themes and provided insight. Relevant actors thought that the profile was presented in an understandable way. They nevertheless indicated that the profile could be improved by making use of information from additional sources (besides the regional public health services and regional primary care support structure). Tables 2, 3 and 4 also shows that actors were less likely to provide data for the profile than the core team. Provision of data (e.g., by primary care professionals) was not possible in all neighbourhoods due to lack of time, and because of privacy or competition concerns. The majority of the core teams felt that compiling and designing the profile cost too much time, but that combining data from public health and primary care was useful. The inclusion of data on the population age structure and prognosis in the profile was considered essential in all neighbourhoods. Comparative regional or national statistics were also considered to be a useful aid to the interpretation of local data.

The organised dialogue was given a good mark by the core teams ( 8 on a scale from 1 to 10 ) and rated satisfactory by the actors (7). The dialogue was thought to be a useful vehicle for discussing issues and solutions and for bringing together people working in the public health and primary care sectors. In some neighbourhoods, the non-involvement of residents or other actors (e.g., GPs) was thought to be a shortcoming. Furthermore, it was not always apparent to the relevant actors how the results were to be used for decision-making and planning (e.g., district plans). For neighbourhoods that were further in the process this was clearer. On the basis of the participants' similar views, a list of important dos and don'ts regarding the use of district health profiles and policy dialogues emerged (see Table 5).

\section{Participants' view of the collaboration process}

The tools were used to initiate collaboration between public health and primary care. The core teams found that the collaboration process was easier to get started in the neighbourhood if there was a clear mandate from the public health sector and primary care sector or if neighbourhood-focused working was already established (formal approach). Figure 3 with relevant aspects for collaboration shows that the core teams were generally a

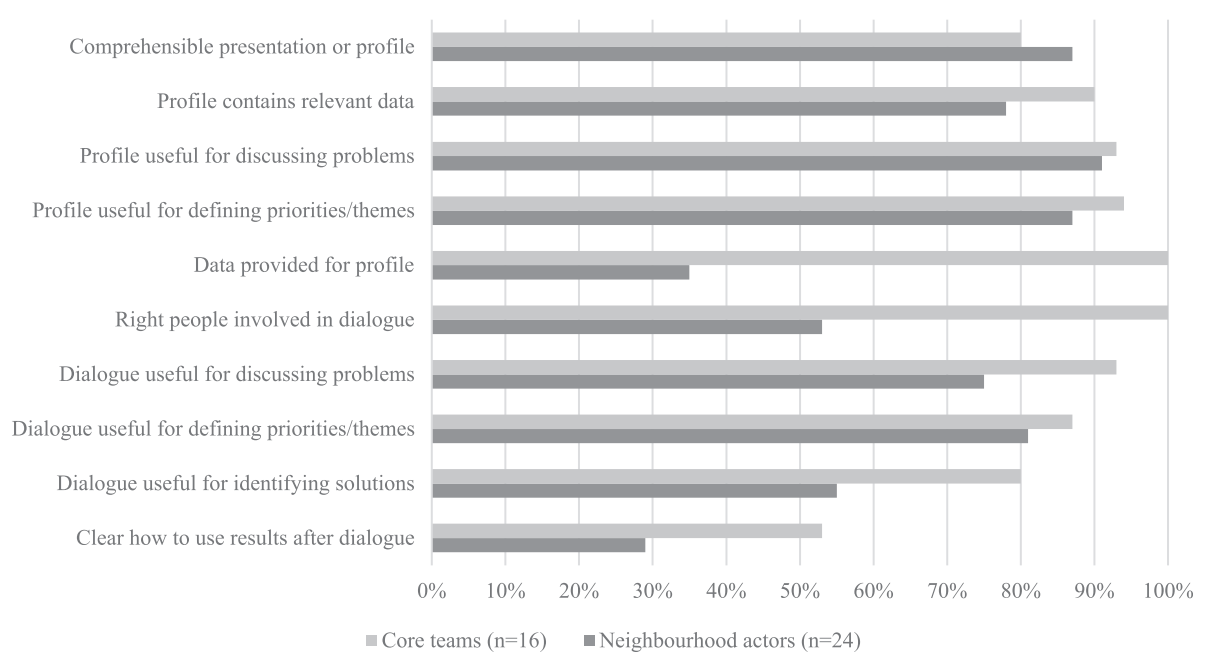

Fig. 2 Views regarding district health profile and policy dialogue 


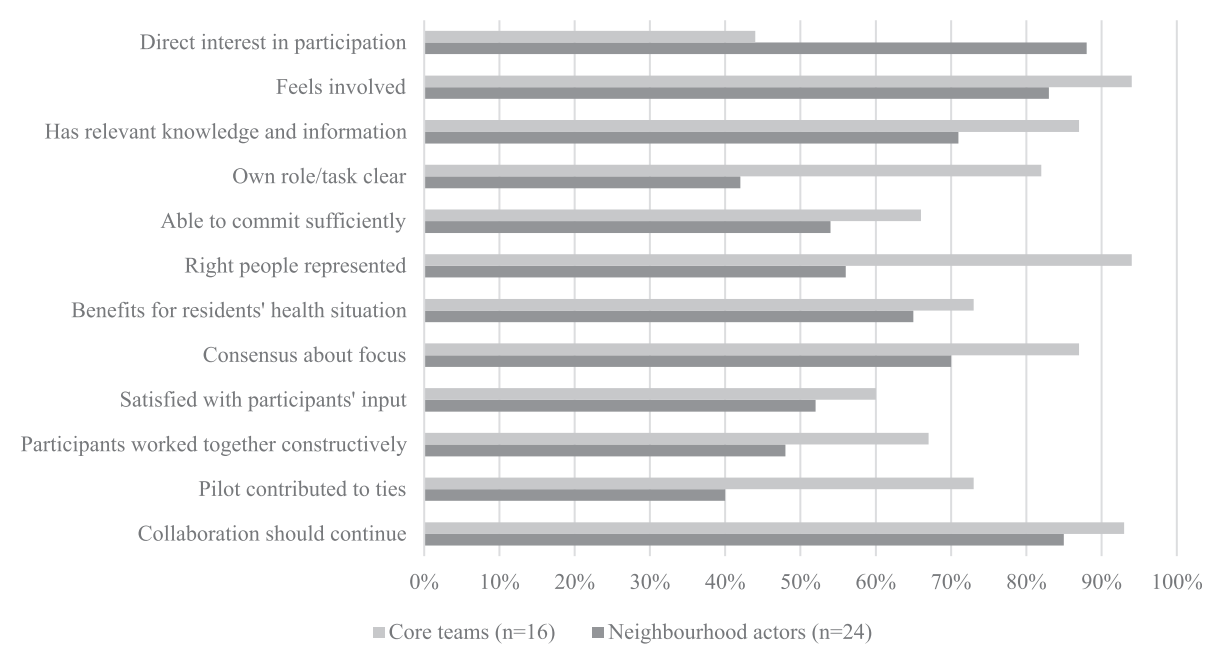

Fig. 3 Views of the collaboration process

Table 5 Dos and don'ts identified from experiences in the seven neighbourhoods

\begin{tabular}{lll}
\hline & Dos & Don'ts \\
\hline District health profiles & $\begin{array}{l}\cdot \text { Do discuss needs with the relevant actors at the outset } \\
\text { and obtain additional data. }\end{array}$ & $\begin{array}{l}\cdot \text { Don't fail to agree on the content of the profile } \\
\text { (general or focused) }\end{array}$
\end{tabular}

- Do include in the profile a description of the local population (demography) and information about how the population age structure is likely to change over time (population prognosis).

- Do use municipal or regional data if no good neighbourhood data are available,. Be open about the data used and discuss how they should be interpreted.

- Do prepare an attractive presentation with a lot of illustrations suitable for the general public. Provide absolute data as well as percentages to help people relate to the information.

Policy dialogues

- Do get to know the local actors before the dialogue and invest time in building relations so that the parties in question do take part.

- Do make a clear choice either for an open dialogue or for a more thematic dialogue.

- Do start the dialogue at an early stage, and consider organising several dialogues using various work forms (e.g., a dialogue with the neighbourhood council at an early stage in order to gauge what residents see as the issues).

- Do keep the organisation of the dialogue under your own control and plan it carefully.

- Do consider holding several dialogues and use various work forms, since one dialogue session is often insufficient

- Do get a councillor, local resident or well-known person to start the dialogue session.

- Do make connections with initiatives already in progress, because there are often a lot of them.

- Do conclude the policy dialogue with definite agreements.
- Don't fail to obtain (sufficient) reliable local data.

- Don't spend too long on data collection, because more or better data can always be found. The profile is a means to an end, not an end in itself.

- Don't fail to allocate enough time to developing the profile.

- Don't fail to allow sufficient opportunity for input from other partners when developing (themes for) the district health profile.

- Don't organise a meeting to select a theme if the theme is already decided (e.g., due to urgency of municipality's needs).

- Don't fail to clearly define the objective of the dialogue.

- Don't organise a dialogue without having sufficient time and funds to make it work.

- Don't use the term 'policy dialogue' when inviting actors (refer to it as a 'neighbourhood dialogue' or 'meeting').

- Don't allow the dialogue to become unstructured, or it will not yield much.

- Don't choose a profile presentation form that is unsuitable for the local actors.

- Don't fail to get important actors (e.g., local residents or GPs) involved, or you will not succeed in bringing people together or forging ties. 
little more positive about the process than the actors. Core teams reported that it took a lot of time to involve local actors in the collaboration process. This is valid for neighbourhoods with little ties between public health and primary care (e.g., no existent working group to join). Most actors had a direct interest in participating in the process and felt engaged. However, their role or task in the process was not always clear or residents' representatives and certain professionals were not involved (e.g., GP's). In neighbourhoods with an engaged centre for primary care or GP, collaboration between GP's and municipalities seem to develop more easily. In most neighbourhoods there was consensus on the focus. However, not in all neighbourhoods there was an alignment between concrete activities of public health and primary care. Although actors indicated that input and connections could be improved, almost all relevant actors from the seven neighbourhoods felt that the collaboration initiated through the process should be continued in order to realise the aims and actions (associated with the themes) in the neighbourhood. That was confirmed by the researchers' observations regarding the organised policy dialogues. A lot of energy and enthusiasm for the idea of getting together and collaborating was generated amongst the various local actors, and there was a desire to continue on similar lines. The insight that problems are amendable if approached from different perspectives contributed to the collaboration between public health and primary care. However, more time is needed to continue and consolidate the collaboration.

\section{Discussion}

Although public health and primary care share the goal of promoting the health and wellbeing of the public, the two health sectors find it difficult to develop mutually integrated plans or policy strategies and to collaborate with each other [8]. The aim of this study was to compare a stepwise process approach implemented in seven neighbourhoods, using district health profiles and policy dialogues as central tools to develop integrated district plans and promote collaboration between public health and primary care.

The study found that participants were in general positive about combining quantitative data (as a result of the profiles) and qualitative data (as a result of the dialogues). In some cases this led to the pointing out of problems which were not detected by looking at the profiles, like illiteracy and poverty. The dialogue could also indicate reasons for further research. The use of different data (stemming from the use of different tools) could support evidence informed policy making [10]. In literature these tools are often studied and described separately, or separate from the process to strengthen intersectoral action $[9,32,33]$. However, in this study a multiple case design was used to develop a practical strategy with attention for both substantive tools as well as the process.

The stepwise approach contains a set of generic process steps, which are applicable on every local situation (e.g., use of own data or network). Differences in the seven neighbourhoods were observed with regard to the operational execution and intensity to completion of the steps. For example, the pre-existence of an active working group (consisting of representatives of the public health sector and primary care sector) or an active GP speed up the process. The sample in this study, although being a convenience sample, contained cases from varying size and location. However, the collaboration does not seem directly related with the size or location of the neighbourhood, but rather with feeling of involvement or consensus on health problems (as relevant aspects for collaboration). Other research shows also that size and location of municipalities are not indicative for the level of intersectoral collaboration [34, 35].

The type of (action) research used in this study required more time investment of researchers, but by developing the strategy in practice it is expected to be transferable. With the aim of the two tools to encouraging more evidence-informed health decision-making, policy development and collaboration in neighbourhoods and getting the practice established in other neighbourhoods in the Netherlands and elsewhere, a number of aspects of the strategy's application require further attention, as discussed below.

\section{Integrated district health profile}

The district health profile is a statistical report on a neighbourhood and its residents, compiled mainly by the core team and staff of the regional public health services (GGD) and regional primary care support structures (ROS). There is a risk that these compilers will not give sufficient attention to including data from other relevant actors, such as the municipality, residents and welfare organisations (e.g., data on poverty, quality of life and social participation). It is important to include data from such actors as well if the public health and primary care sectors are to be brought closer together [20]. A profile has greater value if professionals and residents recognise themselves in it and are able to use it. The data collection should also be aligned with the current information need in practice. Furthermore, links with the social domain provides opportunities, as recent decentralizations led to new municipal tasks and responsibilities to keep local people in good health and actively involved in society (e.g., stay employed, broad participation). Also, it is expected that municipalities are able to provide active support where require and services in a more efficiently [36]. In many European countries the 
movement of decentralizations is visible. Decentralizations has been seen as an integral part of broader health reforms to achieve improved equity, efficiency, quality and financial soundness [37]. Localities make decisions that achieve these improvements (e.g., healthy communities, equity and access). This requires information to support in these new tasks and responsibilities [38, 39]. The quality of the data also requires attention, because good quality data is not always available at the neighbourhood level or is not free (open data sources). Experience in other countries also emphasises that reliable data are essential for establishing a good process at the local level [40]. In that context, there is also a role for organisations at the national and regional levels (e.g., ministry, knowledge institutes, and health insurers).

\section{Organised dialogue with various parties}

The dialogue that serves to integrate the data in the profile with the local actors' knowledge and experience is (in this study) also organised by the core team. Interpretation and joint 'diagnosis of the neighbourhood' (community diagnosis) is essential [11]. There is a risk that insufficient attention will be devoted to the appropriate actors, and important input not therefore secured (e.g., GPs and neighbourhood residents). People living in the neighbourhood need to have the opportunity to say what they perceive to be the biggest issue in the neighbourhood (e.g., lifestyle, youth care, unemployment) [41]. Although local residents play a vital role in future health, prevention and primary care in the neighbourhood, their involvement in the dialogue cannot be taken for granted [42]. The dialogue process has added value if practical action is linked to the results [43]. In a number of neighbourhoods, it was not always clear what followup action was to be taken. However, that may be because participants were asked about the follow-up soon after the dialogue had taken place (during step 7), at which time the core teams were still processing the results and giving feedback to local actors (dialogue summary circulation). If the dialogue is not concluded with clear arrangements being made, ties may not be forged between the public health and primary care sectors, and collaboration may not follow. Such collaboration is important for ensuring that the policy or activities of the various sectors and actors (e.g., municipality, primary care, welfare organisations) actually have a positive influence on the health of the local population [34]. Other research has found that actors (and residents) who have participated in interaction and dialogue are more likely to accept and implement decisions [11, 44].

\section{Stepwise approach in the neighbourhood}

The stepwise approach of forging ties between the public health and primary care sectors is supervised by the core team. The core teams were supported by researchers and 'inspiration days', so conditions were optimally cut to develop the processes in these neighbourhoods. There is a risk that the process in the neighbourhood stops once the profile has been compiled and the dialogue underpinning the neighbourhood plans and activities has been held. Although the process created a healthy basis for progress in the seven neighbourhoods, it is only really a starting point. A sense of engagement may have been created, but proper ties (e.g., shared responsibility) often still need to be created; it is important that sufficient time and attention are devoted to that aim [45]. Hence, the process at the heart of the method described in this article needs to be translated into a more programmatic joint approach to form ties between public health and primary care. It is therefore important to specify how the plans and actions resulting from the process are to be implemented in the neighbourhood, to define the associated responsibilities and to arrange funding. Thus, relations should be established with the municipalities and health insurers at the start of the process, e.g., through neighbourhood development plans or social neighbourhood teams. Other research into integrated health policy (also sometimes cited as 'health in all policies') shows that collaboration is often confined to temporary (subsidised) projects; attention needs to be given to avoiding that pitfall $[44,46]$. In this study, supervision from the regional public health services and primary care support structures worked well, and a similar model could be used in the future [47]. Representatives of these organisations can take on the task of making a joint analysis and initiating a policy dialogue $[9,48]$. Important conditions to start are clear mandate, available resources and right capabilities [9].

\section{Collaboration between public health and primary care}

The duration of the study reported here (roughly a year) was insufficient to allow for the evaluation of plan implementation or collaboration. More formalised approaches, including policies, are needed to support and endorse collaboration between the public health and primary care sectors. Nevertheless, it was observed that collaboration between the regional public health services and primary care support structure improved considerably, and not merely in the fields considered by the study. Because the multiple-case study has its origins in those organisations, they may be more positive about the resulting collaboration than, for example, municipalities, GPs or residents. The supervisory role played by the regional public health services and primary care support structures inevitably means that they were more closely involved with the process and accepted shared responsibility for it. It is therefore important that they commit other actors. The study also showed that local 
actors are open to collaboration and willing to commit to ongoing collaboration around jointly defined themes, such as obesity, social cohesion and loneliness. The same themes have been identified as significant at local level in other countries too [46]. Given that collaboration between the public health and primary care sectors at the operational or local level is still in its process of development, it is important to monitor whether plans are actually implemented and collaboration continued in the future (e.g., drivers and barriers). Collaboration can be seen as an iterative process. Ongoing monitoring of the collaboration started in the neighbourhoods is important to ensure an effective continuous quality improvement process [8]. Progress on the path towards integration will promote the health and wellbeing of the local population.

\section{Conclusion}

This study showed that the stepwise approach based on the combined use of integrated district health profiles and policy dialogues serves as a vehicle for strengthening collaboration (e.g., more closely involved, consensus about focus) between the public health and primary care sectors at the local level. It also leads to integrated health plans or activities aimed at improving the health of the local population (e.g., promotion of healthy lifestyle or participation community). Monitoring is important to observe whether plans are actually implemented and collaboration is continued in the future. Local differences within the stepwise approach may arise in the intensity and form of the various steps, but because they are practical and clearly defined based on local experiences, they remain transferrable to other neighbourhoods (independent of location and size).

\section{Abbreviations}

GGD: Regional public health services; ROS: Regional primary care support structure; GP: general practitioner; VAAM: Supply and Demand Analysis Monitor; ABF: Neighbourhood scan; LINH: Netherlands Information Network of GP database; CBS: Statistics Netherlands; SCP: The National Institute for Social Research; RIVM: National Institute for Public Health and the Environment.
}

\section{Competing interests}

The authors declare that they have no competing interests.

\begin{abstract}
Authors' contributions
IS and AvG conceived and coordinated the multiple case study, participated as case study researchers, carried out the qualitative interviews, questionnaires and observations, developed the stepwise approach, conducted data analyses and prepared the first draft of the manuscript. HvO and IvdG provided advice during the multiple case study and comment the manuscript critically. All authors contributed to and approved of the final version of the manuscript.
\end{abstract}

\section{Authors' information}

IS is a senior researcher in the field of Health in all policies/Integral health policy. She works at the Centre for Health and Society at National Institute of Public Health and Environment (RIVM) and was part-time seconded at Tilburg University/ Tranzo Scientific Center for Care and Welfare. AvG is an epidemiologist at the Regional Public Health Services Brabant-Zuidoost (GGD BZO) and was part-time seconded at Tilburg University/Tranzo Scientific Center for Care and Welfare. HvO is Chief Science Officer Health System Assessment and Policy Support at RIVM and a professor in Public Health at Tilburg University/Tranzo Scientific Center for Care and Welfare. IvdG is a professor in Effectiveness Individual Prevention at Tilburg University/Tranzo Scientific Center for Care and Welfare.

\section{Acknowledgments}

The authors thank the core teams of the seven neighbourhoods, the project group and the steering committee for their valuable contributions to and input regarding this multiple-case study.

This research was funded by The Netherlands Organisation for Health Research and Development (ZONMW, Grant no 204000027).

\section{Transparency}

The lead author (IS) affirms that the manuscript is an honest, accurate, and transparent account of the study being reported; that no important aspects of the study have been omitted; and that any discrepancies from the study as planned have been explained.

\section{Author details}

${ }^{1}$ National Institute for Public Health and the Environment, Centre for Health and Society, PO Box 13720 BA, Bilthoven, The Netherlands. ${ }^{2}$ Regional Public Health Service Brabant-Zuidoost, PO 8684, 5605 KR, Eindhoven, The Netherlands. ${ }^{3}$ Tilburg University, Tranzo Scientific Center for Care and Welfare, PO 90153, 5000 LE, Tilburg, The Netherlands. ${ }^{4}$ National Institute for Public Health and the Environment, Executive Office, PO Box 13720 BA, Bilthoven, The Netherlands.

Received: 25 February 2015 Accepted: 22 September 2015 Published online: 28 September 2015

\section{References}

1. WWS. Gezondheid dichtbij. Landelijke nota gezondheidsbeleid (Health Nearby. National health policy document). The Hague: Ministry of Health, Welfare and Sport; 2011

2. ZonMw. Tools ter bevordering van een effectieve samenwerking preventie en curatie in de wijk (Tools for promoting effective collaboration between the public health and curative care disciplines in the neighbourhood.) http://www.zonmw.nl/nl/projecten/project-detail/tools-ter-bevordering-vaneen-effectieve-samenwerking-preventie-curatie-in-de-wijk-modules-voor-wij/ samenvatting/. Accessed March 2012.

3. WHO. Health 2020. A European policy framework and strategy for the 21 st century. Copenhagen: World Health Organization Regional Office for Europe; 2013.

4. WWS. Zorg en ondersteuning in de buurt. Beleidsbrief (Care and support in the neighbourhood. Policy document). The Hague: Ministry of Health, Welfare and Sport; 2011.

5. BZK. Werken aan wijken. Deel 1. Ervaringen en vuistregels uit het experiment Samenhang in de wijk (Working on neighbourhoods. Part 1. Experiences and rules of thumb from the experiment Cohesion in the neighbourhood). The Hague: Ministry of the Interior and Kingdom Relations; 2011.

6. Kickbusch I, Gleicher D. Governance for health in the 21st century: a study conducted for the WHO Regional Office for Europe. Copenhagen: WHO Regional Office for Europe; 2011.

7. Droomers M, Harting J, Jongeneel-Grimen B, Rutten L, van Kats J, Stronks K. Area-based interventions to ameliorate deprived Dutch neighborhoods in practice: Does the Dutch District Approach address the social determinants of health to such an extent that future health impacts may be expected? Prev Med. 2014;61:122-7.

8. Valaitis R. Strengthening Primary Health Care through Primary Care and Public Health Collaboration Final Report for CFHI. Ottawa: Canadian Foundation for Healthcare Improvement (CFHI); 2012.

9. Levesque J-F, Breton M, Senn N, Levesque P, Bergeron P, Roy DA. The interaction of public health and primary care: functional roles and organizational models that bridge individual and population perspectives. Public Health Rev. 2013;35:1-27(No 1).

10. Lavis J, Boyko JA, Oxman AD, Lewin S, Fretheim A. SUPPORT Tools for evidence-informed health policy making (STP) 14: Organising and using policy dialogues to support evidence-informed policy making. Health Res Policy Syst. 2009;7 Suppl 1:S14. 
11. Cuyler A, Lomas J. Deliberative processes and evidence informed decision making in healthcare: do they work and how might we know? Evidence Policy. 2006;2(3):357-71.

12. Hogendorp J, de Boer A, van Driesten G. Huisarts en gemeenten. Samenwerken in de wijk (GPs and municipalities. Collaboration in the neighbourhood). Utrecht: National General Practitioners' Association (LHV); 2013.

13. ROS Robuust. ROS neighbourhood scan. http://www.ros-netwerk.nl/thema/ geintegreerde-gebiedsanalyse/. Accessed May 2013.

14. Jan van Es Instituut. Wijk- en praktijkscan (Neighbourhood and practice scan). http://www.jvei.nl/wijk-en-praktijkscan/. Accessed May 2013.

15. GG \& GD Utrecht. District health profile. http://www.utrecht.nl/overvolksgezondheid/publicaties-en-onderzoek/onderzoekspublicaties/. Utrecht. Accessed June 2013.

16. Terpstra J, Moerman M. Doorontwikkelen van een wijkprofiel. AMPHI project: proces en resultaat van de ontwikkeling van een gezondheidsprofiel (Further development of a district health profile. AMPHI project: process and outcome of the development of a health profile). Nijmegen: Nijmegen Region GGD and Rivierenland GGD; 2013.

17. van Bon-Martens MJH, de Bakker D, van Loon AJM, de Graaf-Ruizendaal WA, Jacobs-van Bruggen MAM, Zwakhals SLN, van Oers JAM. Afstemming regionale VTV en VAAM. Haalbaarheidsstudie naar een samenhangende informatievoorziening over gezondheid en zorg op lokaal en regionaal niveau (Coordination of regional VTV and VAAM. Feasibility study into the coherent provision of information about health and care at the local and regional levels). TSG 2012; 5: 305-311.

18. Petts J. Barriers to participation and deliberation in risk decisions: evidence from waste management. J Risk Res. 2004;7(2):115-33.

19. Lomas J, Culyer T, McCutcheon C, McAuley L, Law S. Conceptualizing and combining evidence for health system guidance. Final Report. Canada: Canadian Health Services Research foundation; 2005.

20. Storm I, van Gestel A. Preventie en zorg verbinden in de praktijk. Wijkgezondheidsprofiel en beleidsdialoog als handvatten (Connecting public health and primary care in practice. District health profile and policy dialogue as tools). Tilburg: Tranzo; 2014.

21. Yin R. Case study research: Design and methods. 3rd ed. Thousand Oaks: Sage; 2003.

22. George A, Bennet A. Case Studies and Theory Development in the Social Sciences. BCSIA Studies in International Security. Cambridge: MIT Press; 2004.

23. Paes M. Wijkgezondheidswerk. Een studie naar 25 jaar wijkgericht werken aan gezondheid in Den Bosch-Oost (Neigbourhood health work. A study of 25 years of neighbourhood-focused health activities in Den Bosch-Oost). 's-Hertogenbosch: Faculteit der Maatschappij- en Gedragswetenschappen, Universiteit van Amsterdam; 2008.

24. Mulder, M. GGD-regio's 2014. In: Volksgezondheid Toekomst Verkenning, Nationale Atlas Volksgezondheid (GGD regions 2014. In: Public Health Status and Forecasts Report, National Health Atlas). https://www.volksgezondhei denzorg.info/. Accessed June 2014.

25. ROS. ROS-netwerk (ROS-network). http://www.ros-netwerk.nl/. Accessed June 2014

26. CBS. Municipalities on 1 January 2014. http://www.cbs.nl/nl-NL/menu/ methoden/classificaties/overzicht/gemeentelijke-indeling/2014/default.htm. Accessed May 2015

27. McMasterHealthForum. Toolkit 2: Policy Dialogues. https:// www.mcmasterhealthforum.org/about-us/our-work/impact-lab/ktpeoverview/toolkit-2-policy-dialogues. Accessed May 2013.

28. Wagemakers A, Koelen MA, Lezwijn J, Vaandrager L. Coordinated action checklist: A tool for partnerships to facilitate and evaluate community health promotion. Global Health Promot. 2010;17(3):17-28.

29. van Bon-Martens MJH. The art of regional public health reporting. Strengthening the knowledge base for local public health policy [dissertation]. Tilburg: Tilburg University; 2011.

30. Bon-Martens MJH. van, Jeeninga W, Eck ECM van, Goor LAM van de, Oers JAM van. Evaluation of the regional Public Health Status and Forecast report (PHSF) in two Dutch regions. Familiarity with use, usefulness, and preconditions. Tijdschrift voor Gezondheidswetenschappen. 2009;87(7):303-10.

31. de Goede J, van Bon-Martens MJH, Mathijssen JJP, Putters K, van Oers JAM. Looking for interaction: quantitative measurement of research utilization by Dutch local health officials. Health Res Policy Syst. 2012;10(9). doi: 10.1186/ 1478-4505-10-9.
32. Hamilton O. Collaboration for evidence-informed decision making. 2010 (Updated 29 April, 2011). http://www.nccmt.ca/registry/view/eng/55.html. Accessed May 2015.

33. Jacobs K, Grafin G, Lenart M. More than just talk: Connecting science and decision making. Environment: Science and Policy for Sustainable Development. 2005;47(9):6-21.

34. Storm I, Harting J, Stronks K, Schuit AJ. Measuring stages of health in all policies on a local level: the applicability of a maturity model. Health Policy. 2014;114:183-91.

35. Steenbakkers $M$, Jansen $M$, Maarse $H$, de Vries N. Challenging Health in All Policies, an action research study in Dutch municipalities. Health Policy. 2012;105(2-3):288-95.

36. Schalk J, Reijnders MAW, Vielvoye R, Kouijzer I, de Jong M. Decentralization in the Netherlands: from blueprints to tailor-made services? The Hague Governance Quarterly. 2014;2(1).

37. Saltman R, Bankauskaite V, Vrangbaek K. Decentralization in health care. World Health Organization on behalf of the European Observatory on Health Systems and Policies. 2007.

38. BZK. Decentralisatiebrief kabinet (Cabinet decentralisation memorandum). The Hague: Ministry of the Interior; 2013.

39. Huber M, Knottnerus JA, Green L, van der Horst H, Jadad AR, Kromhout D, et al. How should we define health? BMJ. 2011;343:D4163.

40. Mansfield CJ, Wilson JL. Community-level data. N C Med J. 2008;69(2):142-5.

41. Kommer K. Samen optrekken met het sociaal domein (Pulling together with the social domain). De eerstelijns (Primary Care) 2013;48-9.

42. Hoeymans N, Kooiker S. Burgers en gezondheid. Themarapport Volksgezondheid Toekomstverkennen 2014 (Local people and health. Public Health Status and Forecasts Report 2014 Theme Report). Bilthoven: RIVM; 2014.

43. McMasterHealthForum. Evidence briefs and stakeholder dialogues. http:// www.mcmasterhealthforum.org/stakeholders/evidence-briefs-andstakeholder-dialogues. Accessed June 2013.

44. Bekker M, Steenbakker M, Storm I, Jansen M. From instruments towards programme for HiAP decision-support? Health Impact Assessment in the Netherlands. In O'Mullan et al. editor. Integrating Health Impact Assessment into the Policy Process: Lessons and Experiences from Around the World. Oxford; 2013.

45. Jansma R, Wetsema K, Beker M. Raak! Effectieve communicatie van informeren tot co-creëren (Striking! Effective communication of informing to co-create). 2010.

46. Storm I. International Policy Overview: Health in All Policies (HIAP) 2012. http://www.nationaalkompas.nl/object_binary/013534_International\%20 Policy\%200verview_HiAP_Mei2012.pdf. Accessed June 2014.

47. Bloten H, Koornstra A. Samenwerking GGD - ROS: sterke schakels in de regionale keten. Notitie ten behoeve van de GGD-Programmaraad OGZ en ROS-klankbordgroep Voorkomen van Zorg (GGD-ROS collaboration: strong links in the regional chain. Memorandum for the GGD Programme Council OGZ and ROS consultation group Prevention of Care. GGD GHOR Netherlands: Utrecht; 2011.

48. Oxman A, Vandvik PO, Lavis JN, Fretheim A, Lewin S. SUPPORT Tools for evidence-informed health policymaking (STP) 2: Improving how your organization supports the use of research evidence to inform policymaking. Health Res Policy Syst. 2009;7(Suppl1):S2.

\section{Submit your next manuscript to BioMed Central and take full advantage of:}

- Convenient online submission

- Thorough peer review

- No space constraints or color figure charges

- Immediate publication on acceptance

- Inclusion in PubMed, CAS, Scopus and Google Scholar

- Research which is freely available for redistribution 